Objectives: To investigate the risk of cancer in TNFi treated PsA patients compared with standardized rates from the general population in Denmark, Finland, Iceland and Sweden.

Methods: TNFi-treated PsA patients were followed from first registration with TNFi-treatment in ARTIS (Sweden), DANBIO (Denmark), ICEBIO (Iceland) or ROB-FIN (Finland) and linked to the national Cancer Registry in each country. Patients with a cancer history prior to start of follow-up were excluded. We investigated the risk of primary cancer among TNFi-treated PsA patients compared with general population cancer rates standardized to age, sex and calendar period within each country. Standardized incidence ratios (SIRs) were estimated for each country and pooled SIRs were subsequently calculated for both any cancer and site-specific cancers of interest

Results: A total of $5218,2039,270$ and 526 patients were registered as ever TNFi-treated in ARTIS, DANBIO, ICEBIO and ROB-FIN, respectively, contributing a total of 44041 patient years of follow-up across all 4 countries.

For all cancers, the SIRs of TNFi-treated patients from ARTIS, DANBIO, ICEBIO and ROB-FIN were 0.94 (0.80 to 1.10$), 0.99$ (0.77 to 1.26), 1.71 (0.88 to 2.99) and 1.28 ( 0.82 to 1.90$)$, respectively. The number of observed and expected cancers and the SIRs of any and selected site-specific cancers are listed in table 1.

Table 1. Standardized incidence ratios (SIR) of TNFi-treated patients from 4 Nordic countries compared with the general population.

\begin{tabular}{lccc}
\hline & $\begin{array}{c}\text { Observed } \\
\text { cancers }\end{array}$ & $\begin{array}{c}\text { Expected } \\
\text { cancers }\end{array}$ & $\begin{array}{c}\text { SIR (95\% confidence } \\
\text { interval) }\end{array}$ \\
\hline All cancers & 282 & 281.6 & $1.00(0.89$ to 1.13$)$ \\
Colorectal & 32 & 26.5 & $1.21(0.85$ to 1.71$)$ \\
Hodgkin's and non-Hodgkin's & 21 & 11.4 & $1.84(1.20$ to 2.82$)$ \\
lymphoma & 20 & 25.4 & $0.79(0.51$ to 1.22$)$ \\
Lung & 20 & 18.6 & $1.07(0.69$ to 1.66$)$ \\
Malignant melanoma & 8 & 6.6 & $1.21(0.60$ to 2.41$)$ \\
Pancreas & 7 & 7.4 & $0.95(0.45$ to 1.99$)$ \\
Brain & 58 & 48.4 & $1.20(0.93$ to 1.55$)$ \\
Female breast & 6 & 9.0 & $0.67(0.30$ to 1.49$)$ \\
Corpus uteri & 34 & 48.8 & $0.70(0.50$ to 0.98$)$ \\
Prostate & & &
\end{tabular}

Only included sites with $>5$ cancer outcomes.

Conclusion: Our results suggest that the overall cancer risk for TNFi-treated PsA patients is not increased compared to the general population. Further analysis of the risk of malignant lymphomas will inform on whether the increased risk we observed is attributed to the PsA disease or treatment with TNFi.

Acknowledgement: This study is funded by FOREUM and NordForsk.

Disclosure of Interests: Christine Ballegaard Speakers bureau: Janssen Pharmaceuticals., Karin Hellgren: None declared, René Cordtz: None declared, Bénédicte Delcoigne: None declared, Björn Gudbjornsson: None declared, Thorvardur Jon Love Consultant for: Received reimbursment from Celgene for speaking about guidelines for the treatment of psoriatic arthritis, Kalle Aaltonen: None declared, Dan Nordström Grant/research support from: MSD, Pfizer, Consultant for: AbbVie, BMS, MSD, Novartis, Roche, Pfizer, UCB, Speakers bureau: Novartis, UCB, Sella Aarrestad Provan Consultant for: Novartis, Speakers bureau: Lilly, Johan Askling Grant/research support from: Karolinska Institutet (JA) has or has had research agreements with the following pharmaceutical companies, mainly in the context of the ATRIS national safety monitoring programme for rheumatology biologicals: Abbvie, BMS, MSD, Eli Lilly, Pfizer, Roche, Samsung Bioepis, and UCB., Consultant for: Karolinska Institutet has received remuneration for JA participating in ad boards arranged by Lilly, Novartis, and Pfizer., Kristian Zobbe: None declared, Lars Erik Kristensen Grant/research support from: UCB, Biogen, Janssen Pharmaceuticals, and Novartis, Consultant for: Consultant for AbbVie, Amgen, Biogen, BMS, Celgene, Eli Lilly, Janssen Pharmaceuticals, MSD, Novartis, Pfizer, Roche, Sanofi, and UCB Pharma., Speakers bureau: Pfizer, AbbVie, Amgen, UCB, BMS, Biogen, MSD, Novartis, Eli Lilly and Company, and Janssen Pharmaceuticals, Lene Dreyer Consultant for: MSD, UCB and Janssen Pharmaceuticals, Speakers bureau: MSD, UCB and Janssen Pharmaceuticals, Speakers bureau: UCB, MSD, Eli Lilly and Janssen Pharmaceuticals.

DOI: 10.1136/annrheumdis-2019-eular.1763

\section{OP0006 \\ IMPROVEMENT OF ENDOTHELIAL DYSFUNCTION AND INFLAMMATION IN PSORIATIC ARTHRITIS IMPROVE - PSA STUDY}

Ashit Syngle ${ }^{1,2}$, Nidhi Garg ${ }^{3}$, Pawan Krishan ${ }^{3}$, Innovators. ${ }^{1}$ Healing Touch City Clinic, Cardio-rheuma, Chandigarh 160015, India; ${ }^{2}$ Fortis Hospital, Mohali, Sahibzada Ajit Singh Nagar, India; ${ }^{3}$ Punjabi University patiala, Department of Pharmaceutical Sciences and Drug Research, Patiala, India

Background: Cardiovascular (CV) disease is leading cause of death in Psoriatic Arthritis $(\mathrm{PsA})^{1}$. Chronic inflammation in $\mathrm{PsA}$ is associated with vascular endothelial dysfunction and enhanced CV risk. However, the therapeutic options to treat this enhanced $\mathrm{CV}$ risk are limited.

Objectives: To investigate the impact of Olmesartan and Rosuvastatin on endo thelial dysfunction and inflammation in PsA.

Methods: 54 PsA patients randomized to receive 24 weeks of treatment with Olmesartan (OLME) (10mg/day, $n=18)$, Rosuvastatin (Rvs) (10mg/day, $n=18)$, and placebo $(P L)(n=18)$ as an adjunct to existing stable antirheumatic drugs. FMD assessed by AngioDefender. EPCs estimated by flow cytometry. Serum nitrite, TBARS, ICAM-1, VCAM-1 and lipids levels estimated at baseline and after treatment. Inflammatory measures included DAS28, DAPSA, ESR and CRP, proinflammatory cytokines. Quality of life and CV 10-year risk (SCORE high risk charts) were estimated using standard tools.

Results: Baseline levels of FMD and EPC population were impaired indicating endothelial dysfunction. Basal concentrations of inflammatory markers, proinflammatory cytokines and markers of endothelial dysfunction were elevated among three groups. After 24 weeks treatment, FMD improved significantly in rosuvastatin and olmesartan group as compared to placebo. \{OLME vs. PL $(p \leq 0.01)$, Rvs vs. PL $(p<0.01)$, Rvs vs. OLME $(p=0.10)\}$ (Fig.1A). EPCs and nitrite (Fig.1B) levels improved significantly in both rosuvastatin and olmesartan groups. Significant reduction found in ICAM-1 after rosuvastatin treatment $(p<0.01)$ where as olmesartan significantly decreased VCAM-1 $(p=0.04)$ and TBAR levels $(p \leq 0.05)$. Both rosuvastatin and olmesartan resulted in significant reductions of DAS28, DAPSA, ESR, CRP, IL-6 (Fig.1C) and TNF(Fig.1D) as compared to placebo. There was a significant reduction in SCORE, HAQ-DI and SF-36 $(\mathrm{PH})$ score after treatment with rosuvastatin and olmesartan.

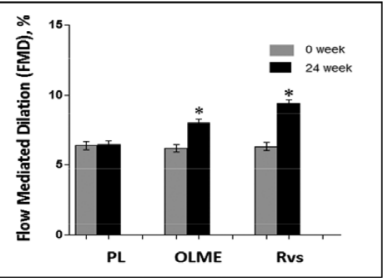

Figure: 1 A Effect of treatment on FMD in PSA.
OOLME vs. PL $(p=0.01)$, Rvs vs. PL ( $p=0.01)$, Rvs vs. OLME (p=0.01)

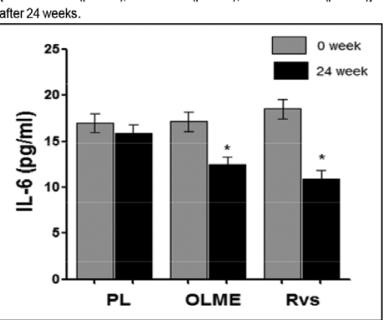

igure: $1 \mathrm{C}$ Effect of trea

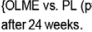$$
\text { 40)\} }
$$
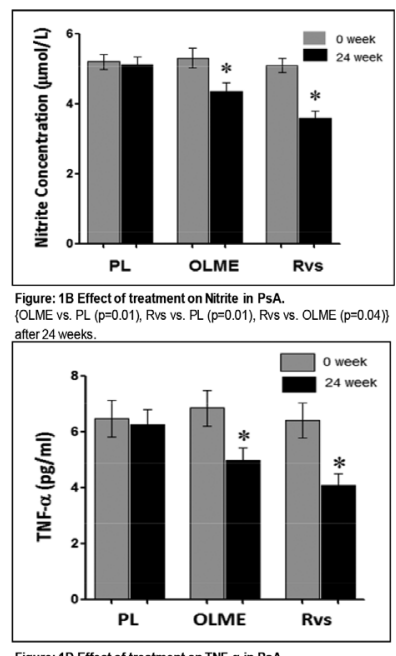

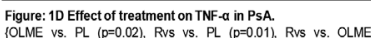

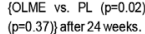

Conclusion: Olmesartan and rosuvastatin improve endothelial dysfunction, inflammation, CV risk and quality of life in PsA patients. Olmesartan and Rosuvastatin lower the proinflammatory cytokines, especially TNF- $\alpha$, that down regulates production of CRP, adhesion molecules and nitric oxide which in turn improves endothelial dysfunction. Both drugs also decrease nitrite concentration and improve the EPC population in PsA patients. The augmentation of EPCs by olmesartan and rosuvastatin represents a fascinating new approach for the management of PsA. However, Rosuvastatin in addition also favourably impacted ICAM-1 and lipid abnormalities. In contrast, olmesartan has beneficial effect on blood pressure. Thus, both rosuvastatin and olmesartan demonstrate immunomodulatory, vasculoprotective and cardioprotective potential in PsA mediated through anti-proinflammatory cytokine action.

\section{REFERENCE:}

[1] Haroon M, Gallagher $P$, Heffernan $E$ and FitzGerald $O$. The Journal of Rheumatology. 2014; $41: 7$

Acknowledgement: None

Disclosure of Interests: None declared

DOI: 10.1136/annrheumdis-2019-eular.5880 